\title{
Histological characterization of the lateral root primordium development in rice
}

\author{
Jun $\mathrm{Ni}^{1}{ }^{12}$, Yan-Xia Shen ${ }^{1}$, Yan-Yan Zhang ${ }^{1}$ and Yu Liư ${ }^{2 *}$
}

\begin{abstract}
Background: Lateral root constitutes an important part of root system either in tap root plants or fibrous root plants. The development of lateral root primordium (LRP) in Arabidopsis, which has a tap root system with simple radial structure of primary root, has been well characterized. However, limited knowledge has been acquired on the plants with fibrous root system, such as rice. This is mainly due to their multiple cell layers coated on root, which disturb the observation of LRP.

Results: We used an easy and quick method to strip the epidermal and cortex tissues of primary root so that the LRP can be easily observed under microscope. In this way, we observed the developmental processes of LRP in rice. In addition, we described the expression dynamics of several root development related genes, especially OsPINs (PIN-FORWMED), during the process of LRP development.
\end{abstract}

Conclusions: We reported an easy and quick method for LRP observation in rice and suggested a "fountain" model of auxin transport in LRP of rice, which is similar with that in Arabidopsis.

Keywords: Rice; Lateral root primordium; Auxin

\section{Background}

Plant root is an important organ for nutrient and water uptake and provides physical support for plant growth. Lateral roots sprout horizontally from the primary root and largely increase surface area of root system to contact with soil. They constitute the major functional part of the root system.

The development of LRP in Arabidopsis has been well documented. The process can be divided into eight stages according to specific anatomical characteristics (Malamy and Benfey 1997). Briefly, the LRP is initiated from the anticlinal divisions of pericycle founder cells (stage I). Then the central short daughter cells divide periclinally to form an inner layer and outer layer (stage II). After series of subsequent divisions (stages III to VII), the LRP finally emerges from the parent root (Casimiro et al. 2003). In the case of cereal plant, such as rice, the situation seems quite different. In rice, the LRP can be initiated from both pericycle and endodermis (Kawata and Shibayama 1965), while in Arabidopsis, only pericycle cells

\footnotetext{
* Correspondence: liuyu_zju@zju.edu.cn

${ }^{2}$ State Key Laboratory of Plant Physiology and Biochemistry, College of Life Science, Zhejiang University, Hangzhou, China

Full list of author information is available at the end of the article
}

contribute to the initiation of LRP (Dubrovsky et al. 2001). In rice, there are several layers of epidermis and ground tissue structure covering the pericycle. They are composed of one layer each of epidermis, exodermis, sclerenchyma and five layers of cortex (Rebouillat et al. 2009). This complex radial structure of primary roots makes it difficult to clearly observe the LRP through microscope.

In this study, we established an easy and quick method to observe the LRP development in rice. For the first time, we described the expression patterns of several developmentally related genes, especially OsPINs, during the process of LRP development. We proposed a "fountain" model of auxin transportation in LRP of rice. In this model, auxin is firstly redirected from parent primary root to the LRP via OsPIN1c, and then transported to the root tip via OsPIN1b, where the auxin is accumulated. This process is proved by the GUS ( $\beta$-glucuronidase) activity of DR5::GUS. In the root tip, part of auxin is retrieved by OsPIN2 mediated auxin transport.

\section{Results and discussion}

Because of the epidermal and the ground tissues, which are composed of exodermis, sclerenchyma, cortex and endodermis, heavily cover the LRP on root of 


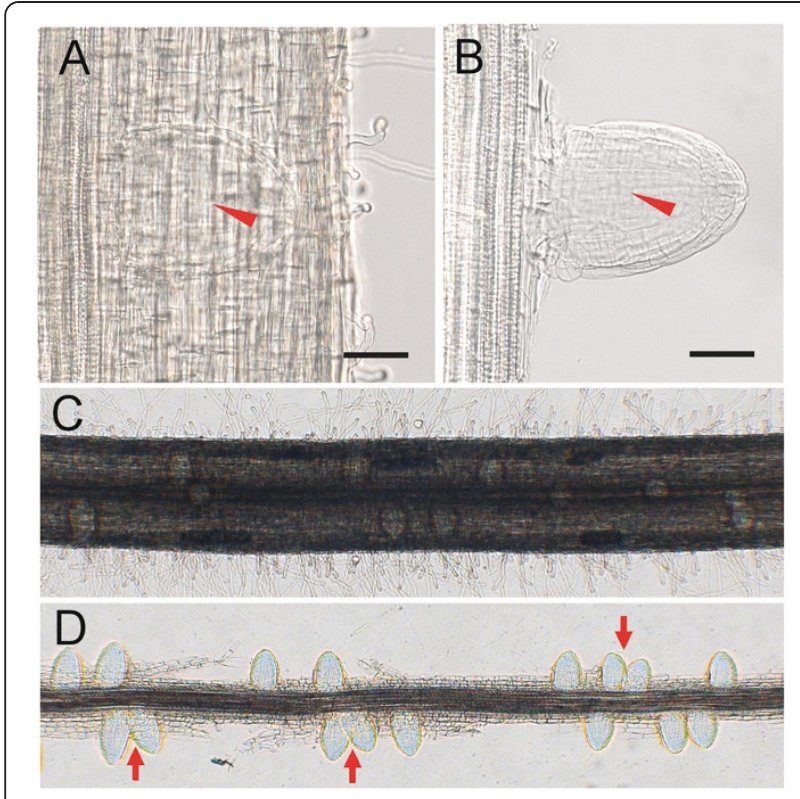

Figure 1 Dissection of the epidermal and ground tissues to uncover the LRP in rice. (A) LRP was covered by epidermal and ground tissues which disturb the observation greatly. Arrow head indicates the LRP, bar $=50 \mu \mathrm{m}$. (B) After dissection of covered layers, the LRP can be clearly observed under the microscope. Arrow head indicates the LRP, bar $=50 \mu \mathrm{m}$. (C) The arrangement of LRPs along the central cylinder cannot clearly seen in normal conditions. (D) After the removal of covered layers, the arrangement pattern and the number of LRPs can be seen clearly. Arrows indicate very closed LRP.

rice (Rebouillat et al. 2009), it is difficult to clearly observe the LRP through microscope (Figure 1A). To cope with this problem, we dissected the epidermal and ground tissues under the stereomicroscope to expose the LRP. In this way, the structure of LRP can be seen clearly under the microscope (Figure 1B). It was relatively hard to exactly count the number of LRPs along a segment of root (Figure 1C). In our experiment, after dissecting the epidermal and ground tissues, we can easily count the number of LRPs. Besides, the distribution pattern of LRPs along the central cylinder can also be observed. In Arabidopsis, the LRPs are allocated along the parent root in a regular left-right alternating pattern (De Smet et al. 2007; Moreno-Risueno et al. 2010). However, there was no obvious left-right alternating pattern observed in rice (Figure 1D). This implies that different regulation systems for LRP initiation exist between rice and Arabidopsis.

\section{The developmental characterization of LRP in rice}

By this method above, the process of LRP development in rice can be clearly observed and characterized. The first scene of LRP initiation observed by this method is the transverse expansion of a specific root tissue, which then formed a dome-shaped LRP (Figure 2A). The domeshaped LRP continues to grow and forms a hemispherical
LRP (Figure 2B,C). After that, the LRP begins to expand transversely to form a shape of mushroom (Figure 2D,E). Then, it grows longitudinally to form a mature lateral root (Figure 2F,G).

To link the process of morphological changes described above to detailed cellular behavior during LRP development, longitudinal sections of LRPs were investigated. At the beginning of LRP development, increased anticlinal divisions are clearly seen in pericycle, resulting in short pericycle cells (Figure 2H). After several rounds of anticlinal divisions in pericycle, periclinal divisions occur in the center of LRP, resulting in two layers, outer layer (OL) and inner layer (IL) as described in Malamy and Benfey (1997). At the same time, the endodermis begins to divide anticlinally which will form a shelter cover the LRP (Figure 2I). The central IL cell divides periclinally, while the OL cell divides anticlinally to form small cuboidal cells. At the same time, the endodermis cells continue to divide anticlinally and the dome shaped LRP begins to appear (Figure 2J). At a certain stage of LRP development, several events appear to occur at approximately the same time. (1) The radial pattern begins to appear and the IL cells develop to form the stele, while the OL form the rest of the tissues. (2) A lens shaped cell appears in the tip of the LRP, which would finally form the root cap of lateral root. (3) The central shelter cells divide periclinally to form two layers of shelter. This shelter will cover the LRP and stop further dividing (Figure $2 \mathrm{~K}$ ). As the LRP continues to develop, LRP begins to resemble the mature lateral root tip. A core of presumptive stellar tissue is surrounded by 4 cell layers corresponding to epidermis, exodermis, sclerenchyma and endodermis as described by Rebouillat et al. (2009). The lens shaped cell also divides to form a potential root cap at the tip of LRP (Figure 2L,M). At the later stages of LRP development, the mushroom shaped primordium can be observed by the methylene blue staining (Figure 2M). At those stages, the cell arrangement of the LRP was very similar to that of the mature lateral root (Figure $2 \mathrm{~N}$ ). These results above showed that, first, the basic process of LRP development in rice is similar to that of Arabidopsis in the cellular level, and more importantly, we connect our morphological descriptions to the cellular organization of LRP which has been well characterized.

\section{Auxin distribution and gene expression patterns in the process of LRP development in rice}

The synthetic auxin responsive promoter DR5 has been used as a tool for monitoring auxin response in planta (Ulmasov et al. 1997). It has been shown that the activity of the reporter correlates to auxin content in roots (Benkova et al. 2003; Casimiro et al. 2001). We examined auxin response during the development of LRP in rice using DR5::GUS transgenic lines. GUS activity was detected at very early stages of development (Figure 3A1). When the 

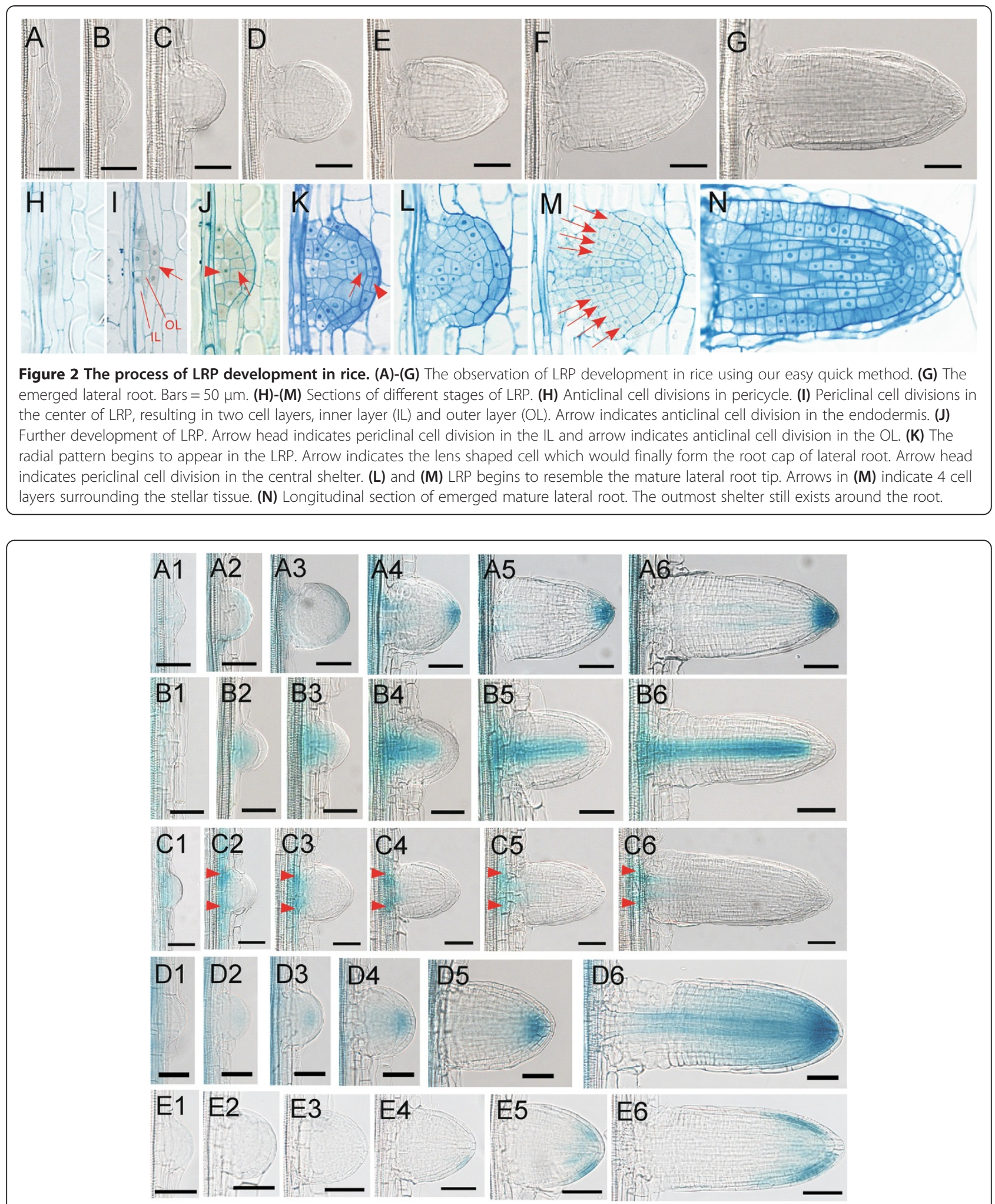

Figure 3 Gene expressions in the process of LRP development in rice. (A1)-(A6) The expressions of DR5::GUS during the development of LRP in rice. (B1)-(B6) The expressions of OsSHR1::GUS during the development of LRP in rice. (C1)-(C6) The expressions of OsPIN1 c::GUS during the development of LRP in rice. Arrow heads indicate the expression peaks at the base of the primordia. (D1)-(D6) The expressions of OsPIN1b::GUS during the development of LRP in rice. (E1)-(E6) the expressions of OsPIN2::GUS during the development of LRP in rice. (A6), (B6), (C6), (D6) and (E6) are the emerged lateral roots. Bars $=50 \mu \mathrm{m}$. 
LRP developed to a hemispherical shape, the GUS staining was observed uniformly in the peripheral of the LRP (Figure 3A2). After that, the GUS staining converged gradually to the central cells in the tip (Figure 3A3). During the progress to later stages, GUS activity was gradually enhanced on the tip of LRP (Figure 3A4-A6). The DR5 activity during LRP development was similar to that in Arabidopsis at least in later stages (Benkova et al. 2003). This indicates that auxin plays similar roles in the development of LRP in rice and Arabidopsis.

OsSHR1 (SHORT-ROOT 1) was reported to express in the stele of primary roots (Cui et al. 2007). We examined its expression in the developing LRPs. OsSHR1 was initially observed to express in the center of hemispherical shaped primordium (Figure 3B2, B3). As long as the LRP growing, OsSHR1 was expressed in the central stele of the primordiun, which was very similar to its expression in the primary root (Figure 3B4-B6). These results also indicated that the identity of stele was existed at the very early stage of LRP development.

OsPIN1c (formerly named OsPIN4) was reported to express in the developing LRP (Wang et al. 2009). We examined its expression in more detail during LRP development based on the method we developed. OsPIN1c was expressed uniformly in the early stage of LRP (Figure 3C1). Gradually, two peaks appeared in the base of LRP and its expression gradually converged to the two peaks (Figure 3C2-C6). From its expression pattern, we proposed that OsPIN1c may function in the junction of LRP and its parent stele to transport auxin between these two parts. We noticed that the two expression peaks were initially found in the boundary of LRP, and later in the central of LRP base, which indicates a transverse expansion in the LRP development.

OsPIN1b was reported to express in the root cap and the stele in the primary root (Wang et al. 2009). We examined its expression in the developing LRP. However, we did not detect the expression of OSPIN1b at the very early stage of LRP development (Figure 3D1). When the LRP developed to a hemispherical shape, a weak GUS activity was detected in the center of LRP, indicating a weak expression of OsPIN1b at this stage (Figure 3D2). As the LRP developed, the GUS staining was clearly detected in the root cap of LRP (Figure 3D3-D5). Interestingly, we did not detect the GUS staining in the stele even though the stele identity was already existed based on the expression patterns of OsSHR1. The expression of OsPIN1b in the stele was not detected until the emergence of LRP (Figure 3D6). At this stage, the expression pattern of OsPIN1b was very similar with that in the primary root (Wang et al. 2009).

OsPIN2 was reported to express in the lateral root cap of primary root (Wang et al. 2009). We examined its expression in the developing LRP. OsPIN2 was not expressed at the early stages of LRP development (Figure 3E1-E3), which was similar to its ortholog PIN2 in Arabidopsis (Benkova et al. 2003). We did not observe the GUS staining until very late stage of LRP development (Figure 3E4, E5). After emergence, OsPIN2 was expressed in margin of the shelter and the root cap in the lateral root, but not in the center of shelter or root cap (Figure 3E6). Its expression pattern in the lateral root was also very similar with that in the primary root (Wang et al. 2009).

In this paper, we reported an easy and quick method for LRP observation in rice. A model was proposed in rice for LRP development. Initially, the LRP expands radically to form a dome shaped LRP. Then the dome shaped LRP further grows to a hemispherical shape. Later the LRP begins to expend transversely to form a shape of mushroom and after that, the LRP grows longitudinally to form a mature lateral root. In addition we also investigated the cellular bases of the morphological changes of LRP. Importantly, using this method, we described the expression dynamic patterns of several genes, especially OsPINs, during the rice LRP development for the first time. The PIN proteins are transporters acting in the efflux of auxin from cells. They have specific developmental roles that largely determined by their highly specific tissue expression (Krecek et al. 2009). AtPIN1 mainly residues at the basal end of the vascular cells in root (Blilou et al. 2005). AtPIN2 localizes apically in epidermal and lateral root cap cells and predominantly basally in cortical cells in the root tip, mediating the auxin maximum and auxin redistribution for root gravitropism (Muller et al. 1998; Blilou et al. 2005). In Arabidopsis, the LRP development was associated with an auxin gradient with a peak at the primordum tip. This gradient depends on differentially expressed PINs (Benkova et al. 2003). In rice, OsPIN1b has been reported to play an important role in crown root emergence and tiller development (Xu et al. 2005). Over-expression of OsPIN2 led to a shorter plant hight, more tillers and a larger angle compared with wild type (Chen et al. 2012). However, the roles of OsPINs in the development of LRP in rice have not been investigated. From the expression patterns of DR5 and OsPINs, we suggested a "fountain" model for auxin transport in the LRP of rice, which was similar to Arabidopsis (Benkova et al. 2003). Auxin is firstly redirected from parent primary root to the LRP via OsPIN1c. Then, auxin transport is mediated by the OsPIN1b to the root tip, where the auxin is accumulated. It can be proved by the staining of DR5::GUS. From here, part of the auxin is retrieved by OsPIN2 mediated auxin transport.

\section{Conclusions}

We reported an easy and quick method for LRP observation in rice and a model was proposed in rice for LRP development. Using this method, we described the 
expression dynamic patterns of several genes, especially OsPINs, during the rice LRP development and suggested a "fountain" model of auxin transport in LRP of rice, which is similar with that in Arabidopsis.

\section{Methods}

\section{Plant growth condition}

Rice seeds (Oryza sativa L. cv. Nipponbare) were grown in solution culture (Yoshida et al. 1976) in controlledenvironment room at temperature regimes of $28 / 22^{\circ} \mathrm{C}$ (day/night) and 70\% humidity under a 12-h photoperiod. One-week-old seedlings were harvest for analysis.

\section{Dissection of epidermal and ground tissues of primary roots}

For dissection of epidermal and ground tissues of rice, the root segments were fixed in FAA (formaldehyde, 95\% ethanol and acetic acid in the volume of 10:85:4) solution for at least 24 hours in $4^{\circ} \mathrm{C}$. After that, the roots were washed in water for several times and operated carefully under the stereomicroscope (Olympus, Japan) with two needles. With longer time of fixation, the LRP can be separated easier. The procedures of staining, dehydration, clearing, infiltration, and embedding were performed according to Liu et al. (2005). The microtome sections were mounted on glass slides for imaging. All the sections showing the patterns of cell divisions in Figure $2 \mathrm{H}-\mathrm{N}$ have been repeated at least three times.

\section{Construction of GUS fusion constructs}

For DR5::GUS vector construction, the DR5 element (Ulmasov et al. 1997) coupled to the CaMV 35S minimal promoter was amplified and digested at the site of Sal I and $B a m \mathrm{H}$ I, and inserted into $p B I 101.3$, which carries the structural gene for GUS and the terminator sequence of the nopaline synthase (NOS) gene. The primers used in PCR amplification were GTCGACGGTATCGCAGCC CCCTTTTGTC and GGATCCTCCCTGTAATGTAA ATAGTAAT. For OsSHR1::GUS vector construction, the promoter of OsSHR1 was amplified by PCR and digested at the site of Hind III and Kpn I, then inserted into pBI101.3. The primers used in PCR were TCAAAGCTTCATAACA TATGGATCAATACAAAC and TCAGGTACCTAAGCA ACGGCGACGAGGAGGA. Construct containing GUS reporter controlled by promoters of OsPIN1c, OsPIN1b and OsPIN2 were constructed before (Wang et al. 2009). These constructs were transformed into the Wild-type (Nipponbare) via Agrobacterium tumefaciens EHA105.

\section{Histochemical analysis and GUS assay}

Histochemical GUS analysis was performed according to Jefferson et al. (1987). Transgenic plant root samples were incubated in $\mathrm{X}$-gluc solution at $37^{\circ} \mathrm{C}$. After staining, the tissues were rinsed and fixed in FAA.

\section{Abbreviations}

LRP: Lateral root primordia; PIN: PIN-FORWMED; OL: Out layer; IL: Inner layer; GUS: $\beta$-glucuronidase; SHR: SHORT-ROOT.

\section{Competing interests}

The authors declare that they have no competing interests.

\section{Authors' contributions}

$J N$, YXS \& YYZ carried out the experiment. JN, YL designed the experiment and drafted the manuscript. All the authors read and approved the final manuscript.

\section{Acknowledgements}

We thank Prof. Ping Wu at Zhejiang for kindly help in this research. This work was supported by grants from the National Basic Research and Development Program of China (2011CB100300), and Zhejiang Provincial Natural Science Foundation of China (NO. LQ13C020005).

\section{Author details}

${ }^{1}$ College of Life and Environmental Sciences, Hangzhou Normal University, Hangzhou, China. ${ }^{2}$ State Key Laboratory of Plant Physiology and

Biochemistry, College of Life Science, Zhejiang University, Hangzhou, China.

Received: 22 November 2012 Accepted: 4 April 2014

Published online: 10 May 2014

\section{References}

Benkova E, Michniewicz M, Sauer M, Teichmann T, Seifertova D, Jurgens G, Friml $J$ (2003) Local, efflux-dependent auxin gradients as a common module for plant organ formation. Cell 115:591-602

Blilou I, Xu J, Wildwater M, Willemsen V, Paponov I, Friml J, Heidstra R, Aida M, Palme K, Scheres B (2005) The PIN auxin efflux facilitator network controls growth and patterning in Arabidopsis roots. Nature 433:39-44

Casimiro I, Marchant A, Bhalerao RP, Beeckman T, Dhooge S, Swarup R, Graham N, Inze D, Sandberg G, Casero PJ, Bennett M (2001) Auxin transport promotes Arabidopsis lateral root initiation. Plant Cell 13:843-852

Casimiro I, Beeckman T, Graham N, Bhalerao R, Zhang H, Casero P, Sandberg G, Bennett MJ (2003) Dissecting Arabidopsis lateral root development. Trends Plant Sci 8:165-171

Chen Y, Fan X, Song W, Zhang Y, Xu G (2012) Over-expression of OsPIN2 leads to increased tiller numbers, angle and shorter plant height through suppression of OsLAZY1. Plant Biotechnol J 10:139-149

Cui H, Levesque MP, Vernoux T, Jung JW, Paquette AJ, Gallagher KL, Wang JY, Blilou I, Scheres B, Benfey PN (2007) An evolutionarily conserved mechanism delimiting SHR movement defines a single layer of endodermis in plants. Science 316:421-425

De Smet I, Tetsumura T, De Rybel B, Frey NF, Laplaze L, Casimiro I, Swarup R, Naudts M, Vanneste S, Audenaert D, Inze D, Bennett MJ, Beeckman T (2007) Auxin-dependent regulation of lateral root positioning in the basal meristem of Arabidopsis. Development 134:681-690

Dubrovsky JG, Rost TL, Colon-Carmona A, Doerner P (2001) Early primordium morphogenesis during lateral root initiation in Arabidopsis thaliana. Planta 214:30-36

Jefferson RA, Kavanagh TA, Bevan MW (1987) GUS fusions: beta-glucuronidase as a sensitive and versatile gene fusion marker in higher plants. EMBO J 6:3901-3907

Kawata S, Shibayama H (1965) On the lateral root primordia formation in the crown roots of rice plants. Proc Crop Sci Soc Jpn 33:423-431

Krecek P, Skupa P, Libus J, Naramoto S, Tejos R, Friml J, Zazimalova E (2009) The PIN-FORMED (PIN) protein family of auxin transporters. Genome Biol 10:249

Liu H, Wang S, Yu X, Yu J, He X, Zhang S, Shou H, Wu P (2005) ARL1, a LOB-domain protein required for adventitious root formation in rice. Plant J 43:47-56

Malamy JE, Benfey PN (1997) Organization and cell differentiation in lateral roots of Arabidopsis thaliana. Development 124:33-44

Moreno-Risueno MA, Van Norman JM, Moreno A, Zhang J, Ahnert SE, Benfey PN (2010) Oscillating gene expression determines competence for periodic Arabidopsis root branching. Science 329:1306-1311

Muller A, Guan C, Galweiler L, Tanzler P, Huijser P, Marchant A, Parry G, Bennett M, Wisman E, Palme K (1998) AtPIN2 defines a locus of Arabidopsis for root gravitropism control. EMBO J 17:6903-6911 
Rebouillat J, Dievart A, Verdeil L, Escoute J, Giese G, Breitler C, Gantet P, Espeout S, Guiderdoni E, Perin C (2009) Molecular genetics of rice root development. Rice 2:15-34

Ulmasov T, Murfett J, Hagen G, Guilfoyle TJ (1997) Aux/IAA proteins repress expression of reporter genes containing natural and highly active synthetic auxin response elements. Plant Cell 9:1963-1971

Wang JR, Hu H, Wang GH, Li J, Chen JY, Wu P (2009) Expression of PIN genes in rice (Oryza sativa L.): tissue specificity and regulation by hormones. Mol Plant 2:823-831

Xu M, Zhu L, Shou H, Wu P (2005) A PIN1 family gene, OsPIN1, involved in auxin-dependent adventitious root emergence and tillering in rice. Plant Cell Physiol 46:1674-1681

Yoshida S, Forno DA, Cock JH, Gomez KA (1976) Laboratory manual for physiological studies of rice, 3rd edn. International Rice Research Institute, Manila, The Philippines

doi:10.1186/s40529-014-0042-x

Cite this article as: Ni et al.: Histological characterization of the lateral root primordium development in rice. Botanical Studies 2014 55:42.

\section{Submit your manuscript to a SpringerOpen ${ }^{\circ}$} journal and benefit from:

- Convenient online submission

- Rigorous peer review

- Immediate publication on acceptance

- Open access: articles freely available online

- High visibility within the field

- Retaining the copyright to your article

Submit your next manuscript at $>$ springeropen.com 